\title{
Relative Prognostic Importance and Optimal Levels of Risk Factors for Mortality and Cardiovascular Outcomes in Type 1 Diabetes Mellitus
}

BACKGROUND: The strength of association and optimal levels for risk factors related to excess risk of death and cardiovascular outcomes in type 1 diabetes mellitus have been sparsely studied.

METHODS: In a national observational cohort study from the Swedish National Diabetes Register from 1998 to 2014, we assessed relative prognostic importance of 17 risk factors for death and cardiovascular outcomes in individuals with type 1 diabetes mellitus. We used Cox regression and machine learning analyses. In addition, we examined optimal cut point levels for glycohemoglobin, systolic blood pressure, and low-density lipoprotein cholesterol. Patients with type 1 diabetes mellitus were followed up until death or study end on December 31, 2013. The primary outcomes were death resulting from all causes, fatal/nonfatal acute myocardial infarction, fatal/nonfatal stroke, and hospitalization for heart failure.

RESULTS: Of 32611 patients with type 1 diabetes mellitus, 1809 (5.5\%) died during follow-up over 10.4 years. The strongest predictors for death and cardiovascular outcomes were glycohemoglobin, albuminuria, duration of diabetes mellitus, systolic blood pressure, and low-density lipoprotein cholesterol. Glycohemoglobin displayed $\approx 2 \%$ higher risk for each $1-\mathrm{mmol} / \mathrm{mol}$ increase (equating to $\approx 22 \%$ per $1 \%$ glycohemoglobin difference), whereas low-density lipoprotein cholesterol was associated with $35 \%$ to $50 \%$ greater risk for each $1-\mathrm{mmol} / \mathrm{L}$ increase. Microalbuminuria or macroalbuminuria was associated with 2 to 4 times greater risk for cardiovascular complications and death. Glycohemoglobin $<53 \mathrm{mmol} / \mathrm{mol}$ (7.0\%), systolic blood pressure $<140 \mathrm{~mm} \mathrm{Hg}$, and low-density lipoprotein cholesterol $<2.5 \mathrm{mmol} / \mathrm{L}$ were associated with significantly lower risk for outcomes observed.

CONCLUSIONS: Glycohemoglobin, albuminuria, duration of diabetes mellitus, systolic blood pressure, and low-density lipoprotein cholesterol appear to be the most important predictors for mortality and cardiovascular outcomes in patients with type 1 diabetes mellitus. Lower levels for glycohemoglobin, systolic blood pressure, and low-density lipoprotein cholesterol than contemporary guideline target levels appear to be associated with significantly lower risk for outcomes.

\author{
Aidin Rawshani, MD, PhD \\ Araz Rawshani, MD, PhD \\ Naveed Sattar, MD, PhD \\ Stefan Franzén, MSc, PhD \\ Darren K. McGuire, MD, \\ MHSC \\ Björn Eliasson, MD, PhD \\ Ann-Marie Svensson, PhD \\ Björn Zethelius, MD, PhD \\ Mervete Miftaraj, MSc \\ Annika Rosengren, MD, \\ PhD \\ Soffia Gudbjörnsdottir, \\ MD, PhD
}

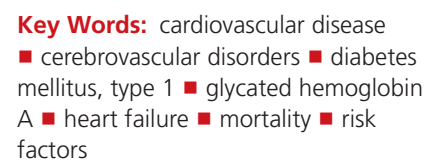

Sources of Funding, see page 1911

(C) 2019 The Authors. Circulation is published on behalf of the American Heart Association, Inc., by Wolters Kluwer Health, Inc. This is an open access article under the terms of the Creative Commons Attribution License, which permits use, distribution, and reproduction in any medium, provided that the original work is properly cited. 


\section{Clinical Perspective}

\section{What Is New?}

- The relative importance of and optimal levels estimated for cardiovascular risk factors for cardiovascular disease and mortality in patients with type 1 diabetes mellitus are sparsely studied.

- We included 32611 patients with type 1 diabetes mellitus; mean follow-up time was 10.4 years, and mean duration of diabetes mellitus was 17.9 years.

- The most important predictors for outcomes were glycohemoglobin, albuminuria, duration of diabetes mellitus, systolic blood pressure, and low-density lipoprotein cholesterol. Lower levels for glycohemoglobin, systolic blood pressure, and low-density lipoprotein cholesterol than contemporary target levels were associated with lower risk for outcomes.

- Albuminuria was associated with 2 to 4 times greater risk for cardiovascular disease and death. Each 1-mmol increase in low-density lipoprotein cholesterol was associated with $35 \%$ to $50 \%$ higher risk for outcomes.

\section{What Are the Clinical Implications?}

- The strongest predictors for mortality and cardiovascular disease in patients with type 1 diabetes mellitus, with the exception of age, were mostly conventional and modifiable cardiometabolic risk factors.

- This suggests that increased clinical focus for these risk factors, particularly in primary prevention, should result in the largest relative risk reduction for mortality and cardiovascular disease.

- Glycated hemoglobin and albuminuria were the 2 most important predictors for mortality and cardiovascular disease in patients with type 1 diabetes mellitus.

- Having lower levels for glycohemoglobin, systolic blood pressure, and low-density lipoprotein cholesterol than recommended in guidelines was associated with a lower risk for myocardial infarction, stroke, and heart failure.

- Future clinical trials could be designed to test our findings.

A ccording to epidemiological studies, individuals with type 1 diabetes mellitus (T1DM) have markedly increased risk for death and cardiovascular outcomes, ${ }^{1}$ whereas dysglycemia is also associated with an increased risk of having additional cardiovascular risk factors in the short or longer term, as well as associated microvascular complications.

The relationship between cardiovascular risk factors and T1DM warrants further investigation, considering the known increased risk for death and cardiovascular complications and the estimated average of $\approx 12$ life-years lost for patients with T1DM from 20 years of age. ${ }^{2}$ Although some management ap- proaches for reducing cardiovascular risk have been extrapolated from randomized trials in type 2 diabetes mellitus, there remains uncertainty beyond optimal glycemia management on how aggressively low-density lipoprotein cholesterol (LDL-C) and blood pressure should be managed. There is therefore interest in improving our understanding of the adverse effects of dysglycemia, the prognostic role of albuminuria, and the role of blood pressure and hypercholesterolemia in patients with T1DM at increased risk for cardiovascular disease. Our research group has previously demonstrated that multiple risk factor control is associated with significantly lower risk for myocardial infarction (MI) and stroke but has little association with risk for heart failure (HF) in individuals with T1DM. ${ }^{3}$ Furthermore, research shows that hyperglycemia, the hallmark of T1DM, even below recommended guideline levels of $6.9 \%$ (or $52 \mathrm{mmol} /$ $\mathrm{mol}$ ) is still associated with at least 2-fold excess risk of death and cardiovascular disease compared with risk in matched control subjects. ${ }^{4}$

In the present study, we applied both conventional and novel statistical approaches to investigate the strength of association of 17 cardiovascular risk factors to illustrate their predictive ability for death and cardiovascular outcomes in individuals with T1DM. In addition, we analyzed the association between varying levels of selected risk factors-glycohemoglobin $\left(\mathrm{HbA}_{1 \mathrm{c}}\right)$, systolic blood pressure (SBP), and LDL-C - to determine optimal risk factor levels with regard to associations with death and cardiovascular outcomes.

\section{METHODS}

The data and analytical methods used for this study will not be made available to other researchers for purposes of reproducing the results or replicating the procedures. The reason for lack of availability is that, by Swedish law that governs national health registries, data sets containing sensitive patient data cannot be shared publicly. Nevertheless, research groups and other health agencies have the option to request data from each government agency, including the National Diabetes Registry.

\section{Study Design and Support}

The Swedish Association of Local Authorities and Regions and other nonprofit agencies supported this study. Commercial sponsorship was not received. The Regional Ethics and Review board of the University of Gothenburg, Sweden, approved the study. All study participants provided written informed consent.

\section{Data Sources and Study Cohort}

The Swedish National Diabetes Register includes information on risk factors, medications, and complications in patients with both T1DM and type 2 diabetes mellitus, presently with $>95 \%$ of all Swedish citizens with T1DM registered. T1DM is 
defined in the National Diabetes Register on the basis of epidemiological criteria: treatment with insulin and a diagnosis at $\leq 30$ years of age, a definition that has been validated as accurate in $>97 \%$ of cases. ${ }^{5}$ Individuals with T1DM and at least 1 registration between January 1, 1998, and December 31, 2012, were included in the study. Information on demographics and clinical characteristics was captured at baseline, that is, the first registration in the registry. Individuals with T1DM who met any of the following criteria were excluded: body mass index $<18.5 \mathrm{~kg} / \mathrm{m}^{2}$, history of coronary heart disease, MI, stroke, HF, atrial fibrillation, severe chronic kidney disease (estimated glomerular filtration rate $[$ eGFR] $\leq 29$ $\mathrm{mL} \cdot \mathrm{min}^{-1} \cdot 1.73 \mathrm{~m}^{-2}$ ), and amputation.

\section{Outcomes}

Outcomes assessed were death resulting from any cause, fatal/ nonfatal $\mathrm{Ml}$, fatal/nonfatal stroke (henceforth referred to as stroke), and hospitalization for HF. Outcomes were retrieved from hospital discharge records with the use of diagnostic codes in the International Classification of Diseases, Ninth Revision and Tenth Revision). The specific codes are listed in Table I in the online-only Data Supplement. The sensitivity and specificity for these outcomes have been validated previously. ${ }^{6}$ Patients were followed up from the index date (first observation in the National Diabetes Register) until death or until December 31, 2013, for all nonfatal outcomes, and until December 31, 2014, for death.

\section{Statistical Analysis}

We applied different statistical approaches, including Cox proportional hazards modeling, analyzing continuous covariates as both continuous and categorical main effects, with machine learning analyses to assess the strength of association for cardiovascular risk factors. We then compared the relative contribution of each predictor in all the models and sorted them according to their relative importance in the models for death and each of the cardiovascular outcomes. All models were adjusted for similar predictors, with the exception of the Cox models, which were stratified by sex to account for different underlying hazards for men and women, with age used as the time scale.

Using 2 developed applications for the interpretation of the Cox proportional hazards model, we determined the relative importance of 17 cardiovascular risk factors in relation to the outcomes of interest. The first statistical approach is called estimated explained relative risk, denoted as $R^{2}$, which measures the uncertainty of the conditional event-free survival time distribution conditional on a set of covariables. ${ }^{7}$ In this method, risk factors are modeled as continuous and categorical covariates as appropriate. As a complementary analysis to the estimated explained relative risk method, the strength of associations was estimated with a second statistical approach developed for Cox proportional hazard models based on the explainable log-likelihood $\left(\chi^{2}\right)$ attributable to each predictor in ANOVA, with categorization of the continuous covariates for this method (Figure 1A-1D). These 2 methods are henceforth referred to as the $R^{2}$ model and the $\chi^{2}$ method.

We also constructed 2 machine learning models (random survival forest and gradient boosting; Figure 2A-2D).
These are both nonparametric ensemble methods that use decision trees, which use bagging and boosting techniques to increase accuracy, to improve robustness, and to reduce variance of predictions. ${ }^{6-8}$ Analysis of right-censored survival data has been implemented into random forest and gradientboosting algorithms. , $^{8,9}$ In addition, we measured the relative contribution of each predictor in every statistical model and analyzed the average percentage for each predictor from all 4 models for death and cardiovascular outcomes (Figure 3). Complementary machine learning models were constructed in which age and duration of diabetes mellitus were omitted from the models to observe the predictors that experienced the greatest relative change in variable importance in the absence of these 2 strong interacting and nonmodifiable predictors. Unstandardized regression coefficients derived from Cox models are presented in Figure 3, next to the predictors in the dot chart.

We used the Cox model with restricted cubic splines and 4 knots for each continuous predictor to delineate the associations between various levels of $\mathrm{HbA}_{1 c^{\prime}} \mathrm{SBP}$, and $\mathrm{LDL}-\mathrm{C}$ and risk for cardiovascular events and mortality (Figure 4A-4D). The evidence-based, guideline-recommended target level was set as reference for each of the 3 risk factors.

Missing data were imputed with the Multivariate Imputation by Chained Equations (MICE) methods. Calculations were performed in $\mathrm{R}$ (version 3.2.3) with the following packages: survival, rms, caret, party, gbm, plotmo, XGBoost, randomForestSRC, ggRandomForest, and mice (Stef van Buuren, Karin Groothuis-Oudshoorn, Alexander Robitzsch, Gerko Vink, Lisa Doove, Shahab Jolani; MICE; R package version $2.25 ; 2015)$. Lists of the variables used in the imputation model are presented in Table II in the onlineonly Data Supplement. Figures I and II in the online-only Data Supplement show the frequency of missing data elements and the distribution of each parameter before and after the imputation.

\section{RESULTS}

\section{Study Population}

A total of 36869 individuals with T1DM were identified. After application of the exclusion criteria, the final cohort comprised 32611 patients (Figure III in the online-only Data Supplement). The Table presents baseline characteristics for all patients with T1DM, for those patients with complete data for all risk factors $(\mathrm{n}=9465)$, and for patients who had at least 1 missing risk factor $(n=23868)$. Baseline characteristics for patients according to different age groups, $\mathrm{HbA}_{1 c}$, blood pressure, and LDL-C levels are presented in Tables III and IV in the online-only Data Supplement. Mean age at entry in the imputed data set was 33.1 years, and $46 \%$ were women. According to the Table, $>60 \%$ of the entire cohort has $\mathrm{HbA}_{1 \mathrm{c}}$ levels $<70 \mathrm{mmol} / \mathrm{mol}$ $(<8.5 \%)$. Median follow-up time was 10.4 years, and mean duration of diabetes mellitus was $17.9 \pm 13.2$ years. A total of 1809 patients (5.5\%) with T1DM died during the study period. 


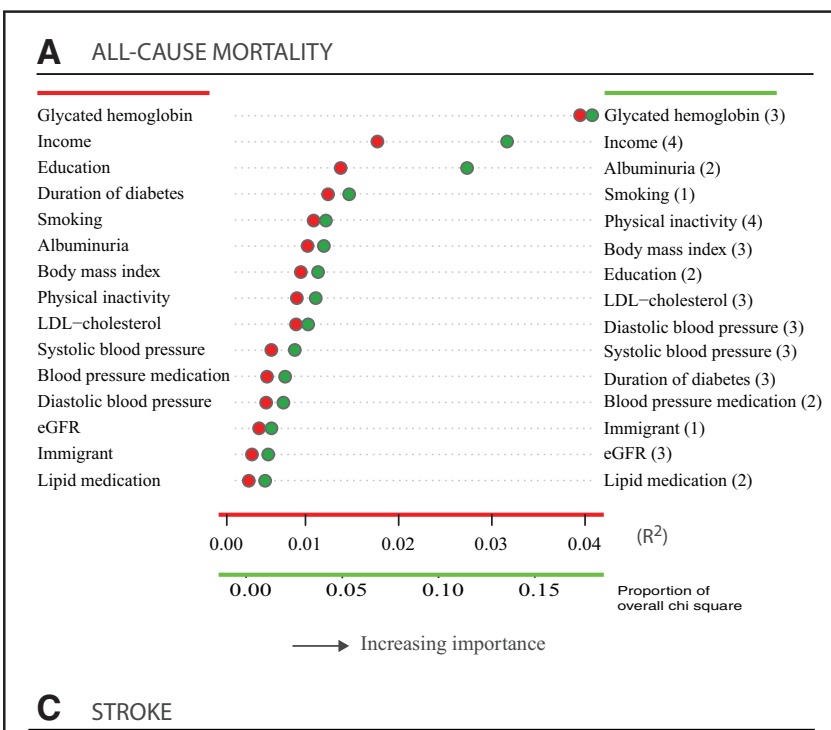

\section{B ACUTE MYOCARDIAL INFARCTION}

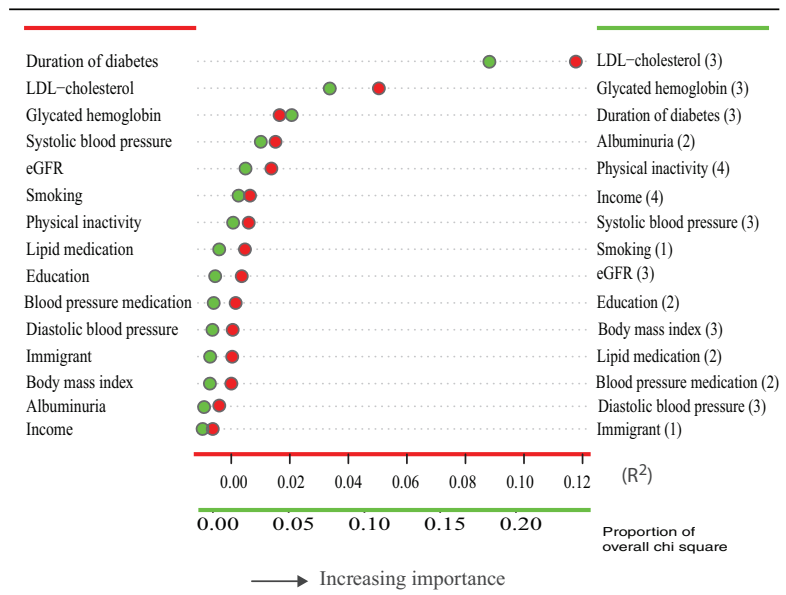

D HEART FAILURE

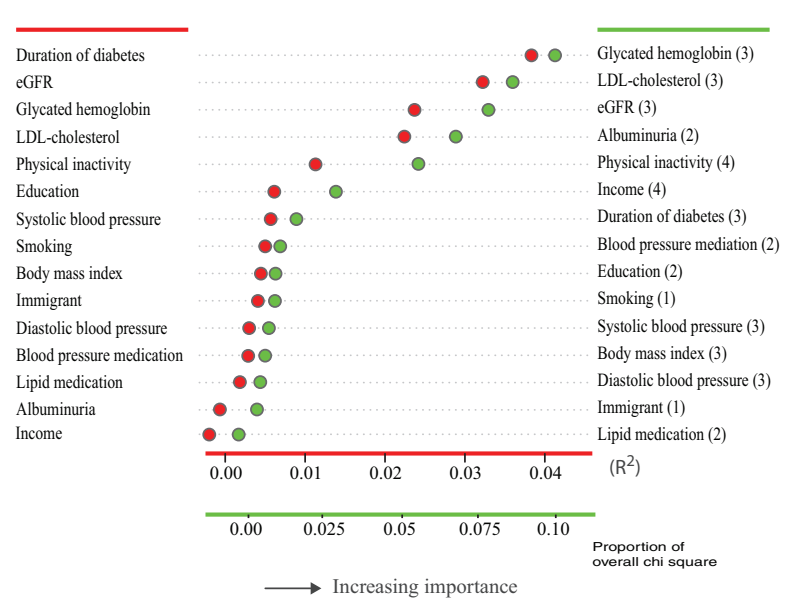

Legend:

Physical inactivity (4)

Glycated hemoglobin (3)

Albuminuria (2)

Systolic blood pressure (3)

LDL-cholesterol (3)

Duration of diabetes (3)

Income (4)

Smoking (1)

Diastolic blood pressure (3)

Education (2)

eGFR (3)

Immigrant (1)

Body mass index (3)

Blood pressure medication (2)

Lipid medication (2)

Immigrant

Income

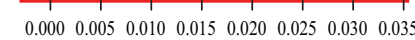

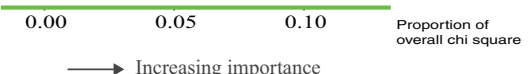

$\longrightarrow$ Increasing importance

$\longrightarrow$ Increasing importance

Cox hazards model ( $\mathrm{R}^{2}$ statistics)

Cox hazards model (explainable log-likelihood, Chi-square). The digits in the parentheses next to risk factors denote number of categories used for each risk factor in the Cox models (number of degrees of freedom).

Figure 1. Strength of association for risk factors according to the estimated explained relative risk model $\left(R^{2}\right)$ and explained log-likelihood for each predictor $\left(\chi^{2}\right)$ among individuals with type 1 diabetes mellitus.

These analyses are based on the same Cox hazards model; we stratified for sex and used age as the time scale. An elevated $R^{2}$ value or overall $\chi^{2}$ suggests that the risk factor has a strong relative importance. The estimated explained relative risk model includes covariables that are modeled as continuous and categorical variables, whereas the $\chi^{2}$ model includes only categories for each predictor. (A) All-cause mortality, (B) nonfatal/fatal acute myocardial infarction, (C) nonfatal/fatal stroke, and (D) hospitalization for heart failure. eGFR indicates estimated glomerular filtration rate; and LDL, low-density lipoprotein.

\section{Strength of Association for Cardiovascular Outcomes and Mortality}

The strengths of association of various risk factors for death and cardiovascular outcomes are presented in Figures 1 and 2A through 2D, showing that age was the strongest predictor for death and each of the cardiovascular outcomes. All risk factors that capture some element of time (eg, age, age at onset of diabetes mellitus, or duration of diabetes mellitus) demonstrated high relative importance measures. Figures 1 and 2A show that beyond age, $\mathrm{HbA}_{1 \mathrm{c}}$ and albuminuria were the strongest predictors for mortality. The Cox model results show that $\mathrm{HbA}_{1 c}$ was by far the most important predictor for death, whereas the machine learning models indicate a complex interaction among age, SBP, $\mathrm{HbA}_{1 c^{\prime}}$ and albuminuria such that these predictors experience significant relative change in variable importance in the context of each of the others. Each 1-mol increase in $\mathrm{HbA}_{1 \mathrm{c}}$ was associated with $2 \%$ higher risk for death (hazard ratio $[\mathrm{HR}], 1.02$ [95\% Cl, 1.017-1.023]; Figure 3), equating to $22 \%(19 \%-26 \%)$ risk difference for each 10 $\mathrm{mmol} / \mathrm{mol}$ increase. Income and SBP were also strong predictors for mortality, with high income associated with $51 \%$ lower risk ( $\mathrm{HR}, 0.49[95 \% \mathrm{Cl}, 0.42-0.59])$ and SBP associated with $0.8 \%$ higher risk (HR, 1.008 [95\% Cl, 1.005-1.012]) per each 1-mm Hg increase, 
A ALL-CAuse MORTALITY

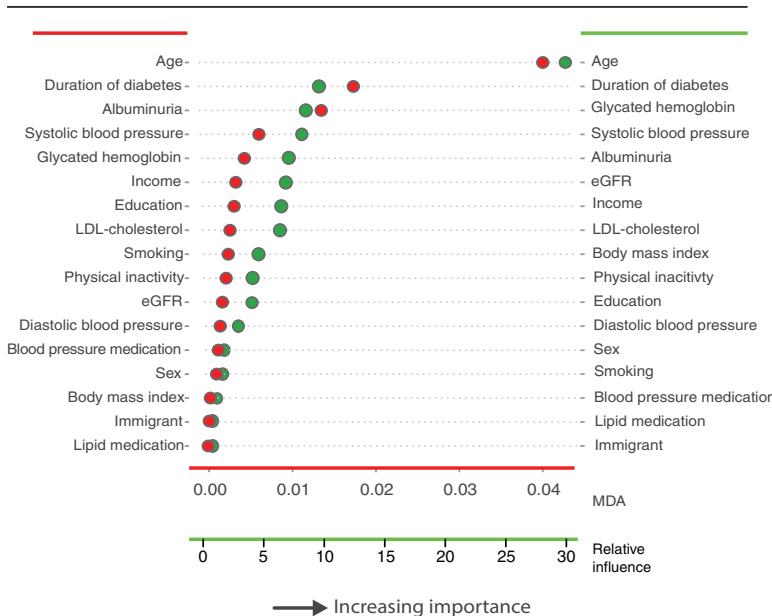

C STROKE

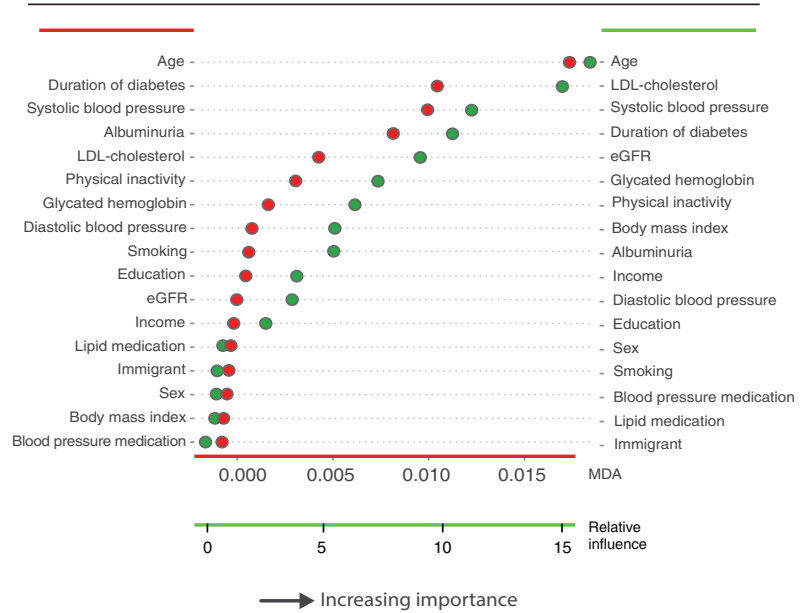

B ACUTE MYOCARDIAL INFARCTION

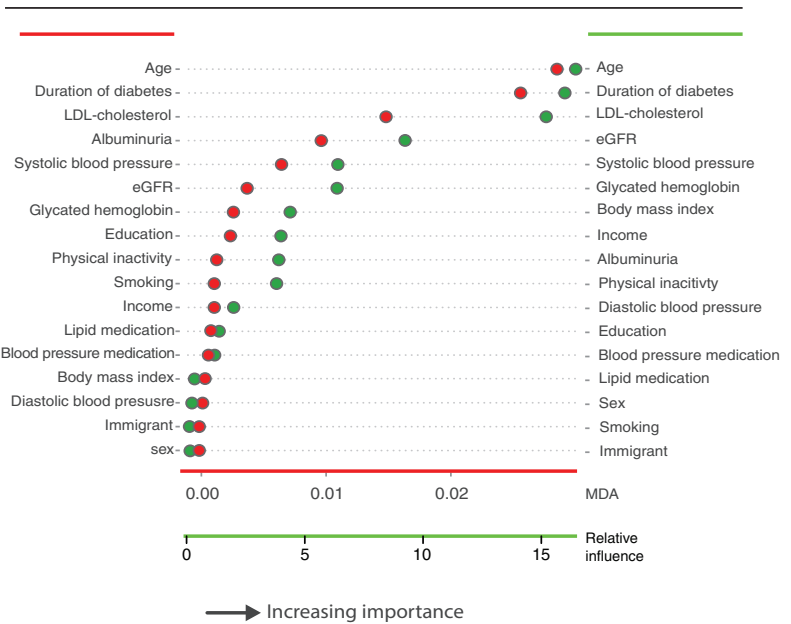

D heART FAILURE

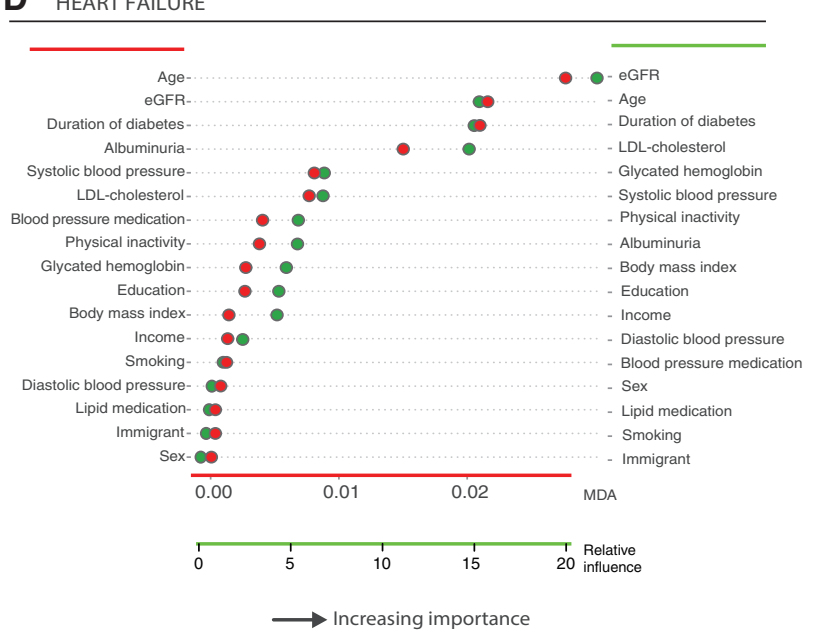

Legend: Random forest (mean-decrease accuracy) - Gradient boosting (relative influence)

Figure 2. Strength of association for risk factors according to the random survival forest (mean decrease-accuracy) and gradient-boosting mode (relative influence).

We constructed a random survival forest and gradient-boosting model to estimate the relative importance for risk factors and outcomes. The machine learning analyses include higher-order interactions between multiple predictors, in contrast to the Cox models. The measure of strength of association for predictors in random forest is denoted as mean decrease of accuracy (MDA) and in gradient boosting as relative influence. Risk factors that display a clear and high MDA or relative influence compared with other adjacent predictors should be considered relevant. (A) All-cause mortality, (B) nonfatal/fatal acute myocardial infarction, (C) nonfatal/fatal stroke, and (D) hospitalization for heart failure. eGFR indicates estimated glomerular filtration rate; and LDL, low-density lipoprotein.

equating to $8.3 \%(5.6 \%-11.2 \%)$ increase per each $10-\mathrm{mm} \mathrm{Hg}$ increase.

Figures 1 and 2B show that duration of diabetes mellitus, LDL-C, and $\mathrm{HbA}_{1 \mathrm{c}}$ were the most important predictors for MI. The machine learning models suggest that duration of diabetes mellitus, albuminuria, and SBP have statistically significant interactions with $\mathrm{HbA}_{1 c^{\circ}}$ Each 1-mmol/L higher LDL-C (38.7 mg/dL) was associated with higher relative risk for $\mathrm{MI}(\mathrm{HR}, 1.47[95 \%$ $\mathrm{Cl}, 1.39-1.55]$; Figure 3). The results for stroke were similar to those for MI, except for SBP and physical inactivity, which proved to be more important predictors for stroke (Figures 1 and 2C). SBP demonstrated a large relative importance compared with other predictors, and the Cox model results showed that each $1-\mathrm{mm} \mathrm{Hg}$ increase in SBP was associated with a 1.5\% higher relative risk (HR, 1.015 [95\% Cl, 1.011-1.02]) for stroke, equating to a $16 \%(12 \%-20 \%)$ risk difference for each $10-\mathrm{mm} \mathrm{Hg}$ rise.

Figures 1 and 2D suggest that eGFR, $\mathrm{HbA}_{1 c}, \mathrm{LDL}-\mathrm{C}$, $\mathrm{SBP}$, and duration of diabetes mellitus were the most important predictors of hospitalization for HF. Comparison between the Cox model results and machine learning analyses demonstrates that age and duration of diabetes mellitus seem to partially account for the association of albuminuria, $\mathrm{SBP}$, and $\mathrm{HbA}_{1 c}$ with $\mathrm{HF}$ 


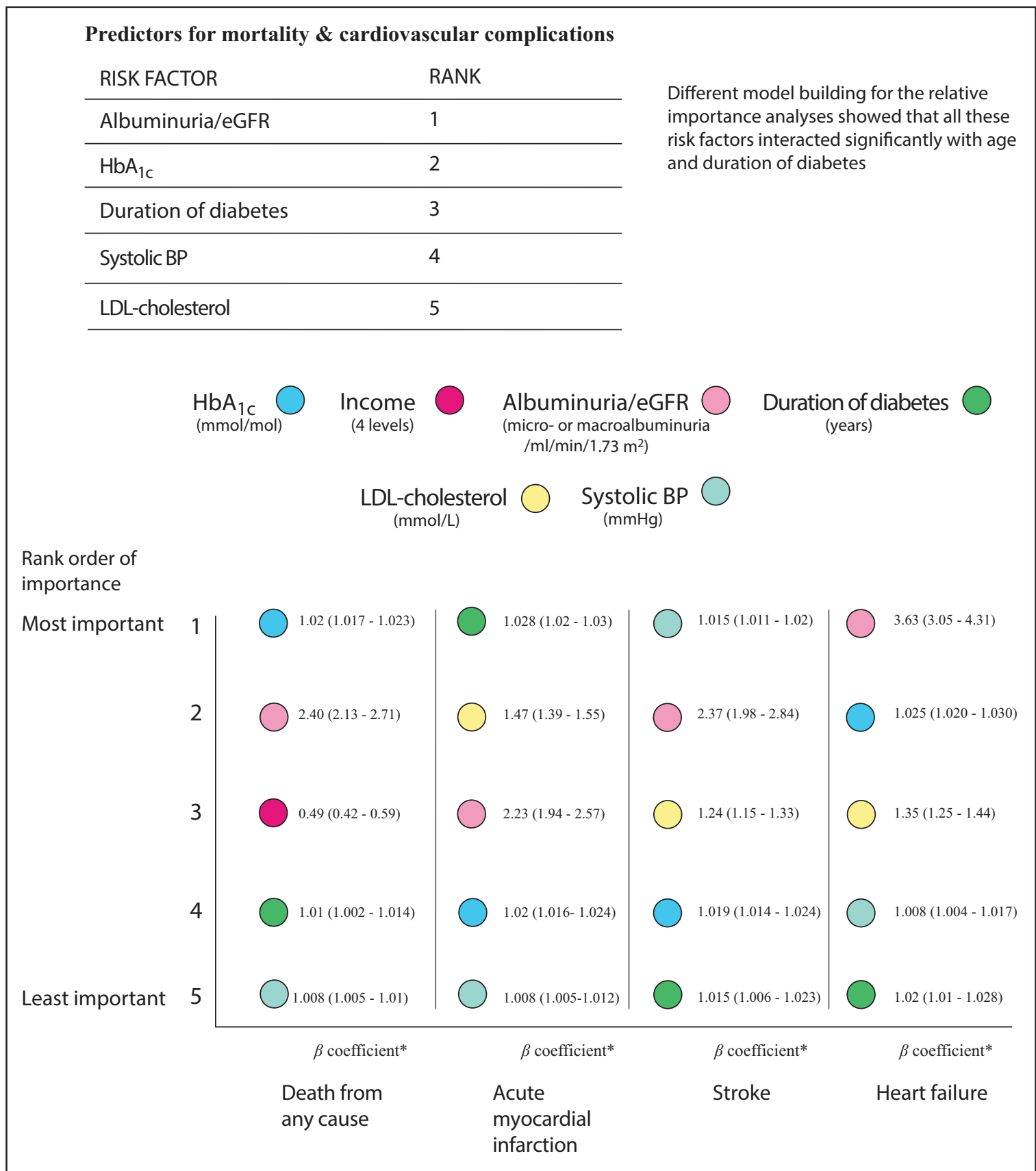

Figure 3. Strength of association for the most important risk factors derived from the Cox models and machine-learning analyses for all-cause mortality, acute myocardial infarction, stroke, and hospitalization for heart failure.

The strength of association for predictors was ranked according to highest relative contribution of each risk factor in every model and outcome. In addition to the relative importance of risk factors, we estimated the unstandardized regression coefficient with the Cox model. Standardized coefficients would enable direct comparison between relative importance of predictors, but this was not possible because albuminuria is a categorical variable. BP indicates blood pressure; eGFR estimated glomerular filtration rate; $\mathrm{HbA}_{1 c^{\prime}}$ glycohemoglobin; and LDL, low-density lipoprotein.

risk. Albuminuria and eGFR (renal function) remained important predictors in the machine learning models, whereas the relative importance of $\mathrm{HbA}_{1 \mathrm{c}}$ was reduced. The HR for albuminuria was $3.63(95 \% \mathrm{Cl}, 3.05-4.31)$.

An illustrative example for Figure 3 follows. From the $R^{2}$ model for mortality, we obtain the $R^{2}$ value for $\mathrm{HbA}_{1 \mathrm{c}}$ $\left(0.043 R^{2}\right)$, divided by the sum of $R^{2}$ for all predictors (0.126 $R^{2}$ value), which translates to $34 \%$ relative contribution in the $R^{2}$ model. The relative contribution of each predictor is also obtained for the $\chi^{2}$, random forest, and gradient-boosting models in a similar fashion. We thereafter calculate the average percentage of the relative contribution from all models and for each outcome. These results are presented in Figure 3 and Table $V$ in the online-only Data Supplement. The relative importance of risk factors, when age and duration of diabetes mellitus were omitted from the machine learning models, is presented in Figure IV in the online-only Data Supplement. 

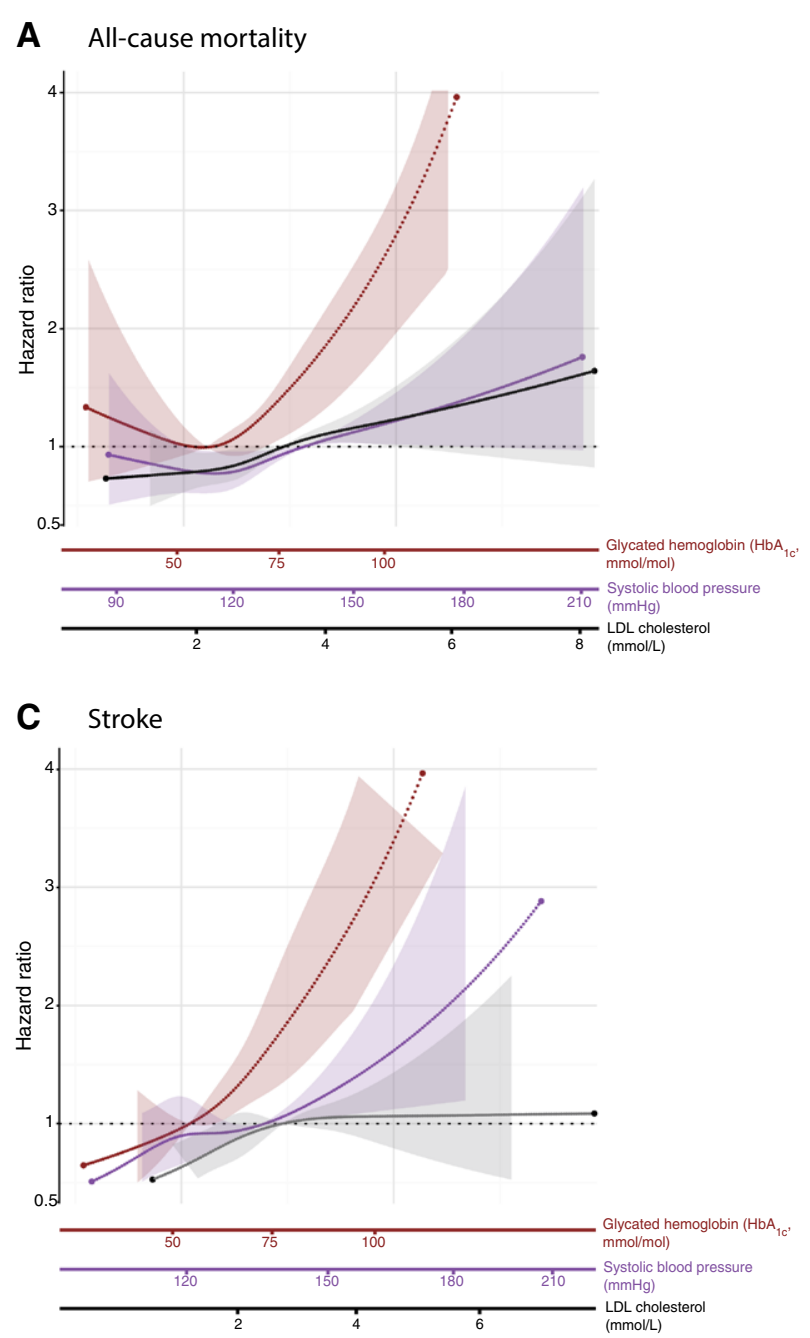

B Acute Myocardial Infarction

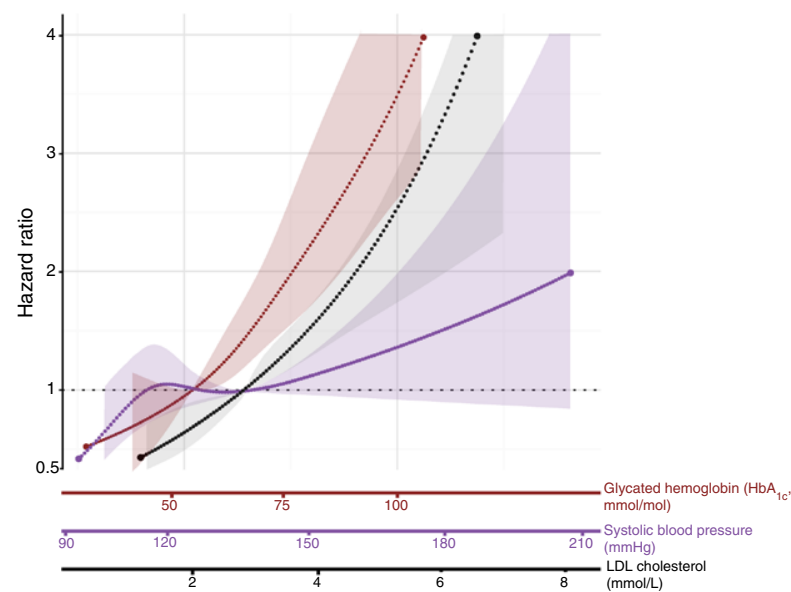

D Heart failure

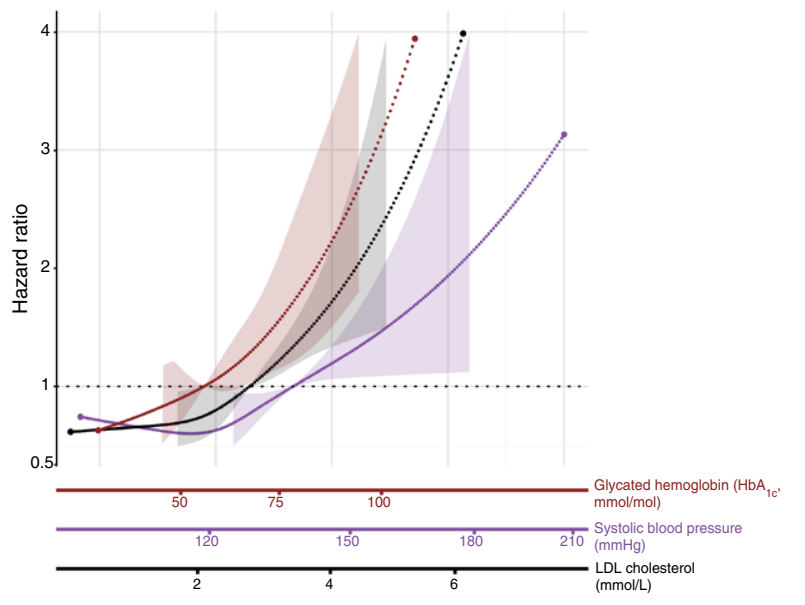

Figure 4. Association between glycohemoglobin $\left(\mathrm{HbA}_{1}\right)$, systolic blood pressure (SBP), and low-density lipoprotein (LDL) cholesterol (LDL-C) and all-cause mortality, acute myocardial infarction (AMI), stroke, and hospitalization for heart failure.

Applying a prediction function to the Cox model allows assessment of the relationship between $\mathrm{HbA}$, SBP, and LDL-C and risk of mortality, AMl, stroke, and heart failure. Continuous variables were modeled with restricted cubic splines, whereas categorical variables were stratified. (A) All-cause mortality, (B) nonfatal/fatal AMI, (C) nonfatal/fatal stroke, and (D) hospitalization for heart failure.

\section{Optimal Levels of Cardiovascular Risk Factors in Patients With T1DM}

Figure 4A through $4 D$ shows the optimal levels for 3 conventional risk factors- $\mathrm{HbA}_{1 c^{\prime}} \mathrm{SBP}$, and LDL-C-for each of the key outcomes of interest. Figure $4 \mathrm{~A}$ shows that the lowest risk of mortality was found at levels of LDL-C and SBP below those recommended by current guidelines, with $\mathrm{HbA}_{1 c}$ displaying an U-shaped relationship. As for Ml, we observed a clear linear association with similar risk trajectories for $\mathrm{HbA}_{1 \mathrm{c}}$ and $\mathrm{LDL}-\mathrm{C}$, whereas SBP displayed a sigmoidal association. Levels below those recommended by guidelines were associated with the lowest risk for $\mathrm{Ml}$ for all 3 risk factors (Figure 4B). Stroke and MI showed similar associations (Figure 4C), except for LDL-C; lipid levels $<2.5 \mathrm{mmol} / \mathrm{L}$ were associated with reduced risk, and elevated levels of LDL-C were not associated with higher risk. For hospitalization for HF (Figure 4D), we observed similar risk trajectories for all 3 risk factors; that is, levels lower than guideline-recommended levels were associated with reduced risk.

\section{Interactions Between Age, Duration of T1DM, and Cardiovascular Risk Factors}

After exhaustive subset research with Cox regression and machine learning models, we observed that age, duration of diabetes mellitus, SBP, $\mathrm{HbA}{ }_{1 c^{\prime}} \mathrm{LDL}-\mathrm{C}$, and albuminuria/eGFR were interacting with each other on different outcomes.

The machine learning models (ie, random survival forest and gradient boosting) have an inherent function to assess complex interaction effects between all predictors. Thus, omitting various risk factors from the machine learning models allowed us to assess how vari- 
Table. Baseline Characteristics for Patients With T1DM According to $\mathrm{HbA}_{1 \mathrm{c}}$ Levels

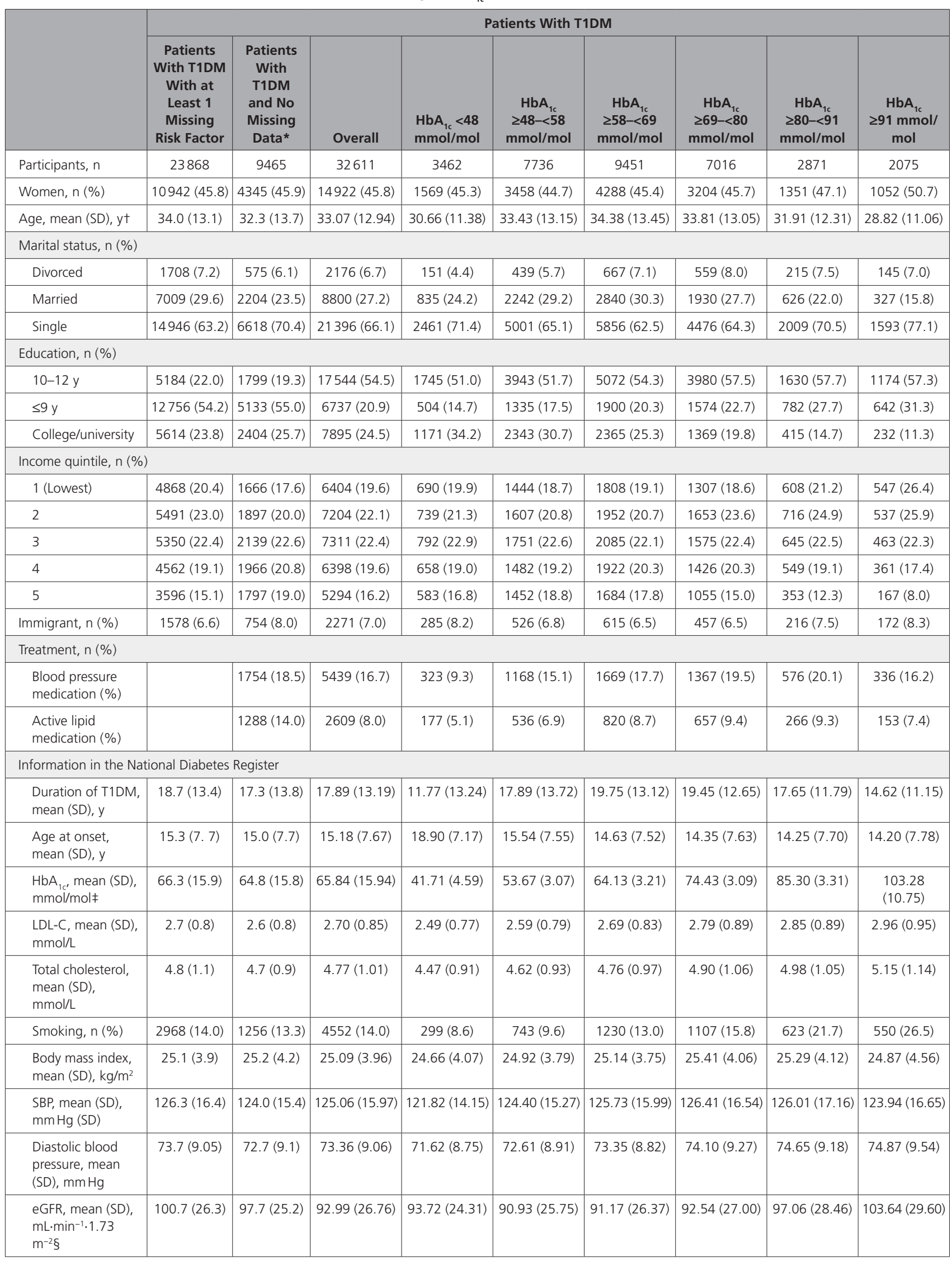


Table. Continued

\begin{tabular}{|c|c|c|c|c|c|c|c|c|c|}
\hline & \multicolumn{9}{|c|}{ Patients With T1DM } \\
\hline & $\begin{array}{l}\text { Patients } \\
\text { With T1DM } \\
\text { With at } \\
\text { Least } 1 \\
\text { Missing } \\
\text { Risk Factor }\end{array}$ & $\begin{array}{l}\text { Patients } \\
\text { With } \\
\text { T1DM } \\
\text { and No } \\
\text { Missing } \\
\text { Data* }\end{array}$ & Overall & $\begin{array}{l}\mathrm{HbA}_{1 \mathrm{c}}<48 \\
\mathrm{mmol} / \mathrm{mol}\end{array}$ & $\begin{array}{c}\mathrm{HbA}_{1 \mathrm{c}} \\
\geq 48-<58 \\
\mathrm{mmol} / \mathrm{mol}\end{array}$ & $\begin{array}{c}\mathrm{HbA}_{1 \mathrm{c}} \\
\geq 58-<69 \\
\mathrm{mmol} / \mathrm{mol}\end{array}$ & $\begin{array}{c}\mathrm{HbA}_{1 \mathrm{c}} \\
269-<80 \\
\mathrm{mmol} / \mathrm{mol}\end{array}$ & $\begin{array}{c}\mathrm{HbA}_{1 \mathrm{c}} \\
\geq 80-<91 \\
\mathrm{mmol} / \mathrm{mol}\end{array}$ & $\begin{array}{c}\mathrm{HbA}_{1 \mathrm{c}} \\
\geq 91 \mathrm{mmol} / \\
\mathrm{mol}\end{array}$ \\
\hline \multicolumn{10}{|l|}{ Albuminuria, n (\%) } \\
\hline No albuminuria & $14336(84.3)$ & $8127(85.9)$ & $28016(85.9)$ & 3252 (93.9) & $6949(89.8)$ & 8081 (85.5) & $5790(82.5)$ & $2306(80.3)$ & $1638(78.9)$ \\
\hline Microalbuminuria & $1440(8.5)$ & $926(9.8)$ & $2791(8.6)$ & $136(3.9)$ & $483(6.2)$ & $880(9.3)$ & $740(10.5)$ & $323(11.3)$ & $229(11.0)$ \\
\hline Macroalbuminuria & $1225(7.2)$ & $412(4.4)$ & $1804(5.5)$ & $74(2.1)$ & 304 (3.9) & $490(5.2)$ & $486(6.9)$ & $242(8.4)$ & $208(10.0)$ \\
\hline
\end{tabular}

eGFR indicates estimated glomerular filtration rate; $\mathrm{HbA}_{1 c^{\prime}}$ glycohemoglobin; LDL-C, low-density lipoprotein cholesterol; SBP, systolic blood pressure; and T1DM, type 1 diabetes mellitus.

*Patients with T1DM and no missing risk factors were a subset of patients with complete data for $\mathrm{HbA}_{1 c^{\prime}} \mathrm{SBP}, \mathrm{LDL}-\mathrm{C}$, albuminuria, and smoking.

†Plus-minus values are mean \pm SD.

$\neq$ Concentrations of $\mathrm{HbA}_{1 \mathrm{c}}$ were based on values from the International Federation of Clinical Chemistry and Laboratory Medicine.

$\S$ The eGFR was estimated with the use of the Modification of Diet in Renal Disease equation.

able importance changed, depending on the risk factor that was excluded from the model and not allowed to interact with other predictors.

The Cox regression and machine learning models showed that age was the strongest risk factor for mortality and cardiovascular disease. Including age in the Cox models appeared to drown out the effect of the remaining variables. This was partially the reason for modeling age as the time scale in the Cox models. This is not possible in the machine learning models; therefore, we were able to observe the effect of age and any interaction effects of age on other predictors. Our machine learning models revealed that age, duration of diabetes mellitus, $\mathrm{HbA}_{1 c^{\prime}} \mathrm{LDL}-\mathrm{C}$, and albuminuria/eGFR were best predicted by each other (Figure 5 provides more information).

After comprehensive model construction with machine learning and Cox models, we observed an interaction effect between the abovementioned variables. Therefore, we constructed 2-way partial dependence plots based on the gradient boosting models to visualize the interacting effect between these variables (Figure 6 provides more information). In conclusion, these analyses indicate that age, duration of diabetes mellitus, SBP, HbA ${ }_{1 c}, \mathrm{LDL}-\mathrm{C}$, and albuminuria/eGFR are the most important predictors for mortality and cardiovascular outcomes. In addition, each of these risk factors appeared to be a strong predictor of each other and seem to interact.

\section{Sensitivity Analyses}

We performed sensitivity analyses to assess the reliability of our models that were based on the imputed data set. Therefore, we constructed 2 cohorts of patients with T1DM. The first cohort included patients with no missing data for $\mathrm{HbA}_{1 \mathrm{c}^{\prime}}$ SBP, LDL-C, albuminuria, and smoking (ie, complete cases, $\mathrm{n}=9465$ pa- tients). The other cohort included patients with at least 1 missing factor ( $n=23868$ patients). We constructed a Cox regression model using explainable log-likelihood explained by each predictor, along with the machine learning method called the gradient-boosting model, for both cohorts to assess mortality and cardiovascular outcomes in patients with T1DM. We did not perform these analyses with the random survival forest model or the explained relative risk model from the Cox model because of excessive processing time from these statistical programs.

The strength of association for patients with T1DM and complete cases or at least 1 missing risk factor is included in the online-only Data Supplement (for more information see Figures V-VIII online-only Data Supplement). The sensitivity analyses demonstrated results comparable to our findings from the imputed data set.

\section{DISCUSSION}

In this report of 32611 patients with T1DM derived from a population-based nationwide cohort, beyond age, the strongest predictors of cardiovascular outcomes and death were $\mathrm{HbA}_{1 c^{\prime}}$ renal function, duration of diabetes mellitus, LDL-C, and SBP. Results also suggest that cardiovascular disease and death represent, as anticipated, somewhat different pathophysiological processes by the variability in the strength of associations of risk factors analyzed in this cohort of relatively young individuals with T1DM. Age was the strongest prognostic factor for all outcomes in both the Cox model and machine learning analyses. With a focus on modifiable factors, $\mathrm{HbA}_{1 c}$ and albuminuria were robust predictors for mortality and hospitalization for $\mathrm{HF}$, in contrast to atherothrombotic disease (ie, MI or stroke), for which conventional cardiometabolic factors such as $\mathrm{HbA}_{1 c^{\prime}} \mathrm{LDL}-\mathrm{C}$, and SBP demonstrated the greatest relative prognostic importance. High income was also as- 


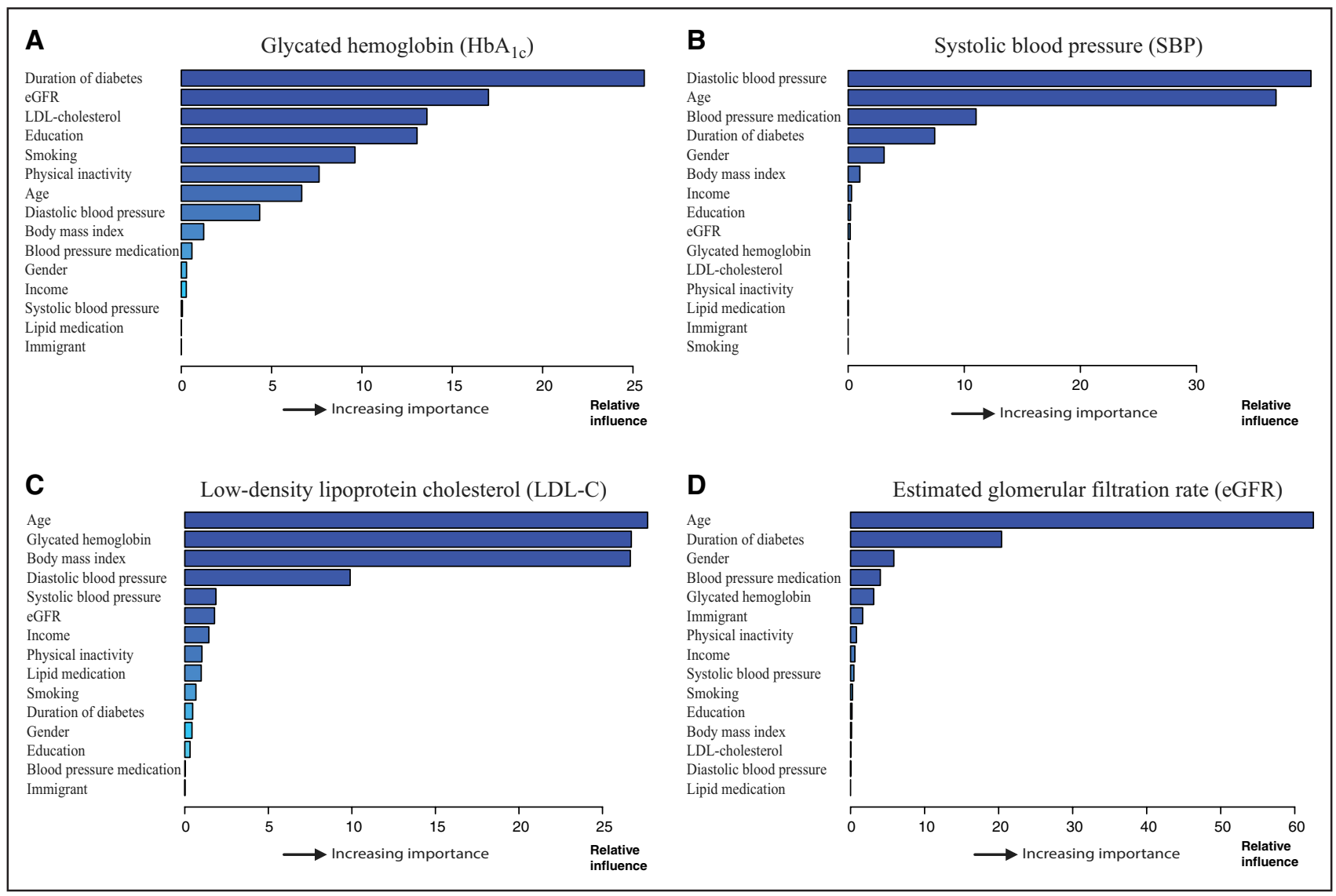

Figure 5. Strength of association of predictors for the selected risk factors glycohemoglobin $\left(\mathrm{HbA}_{1}\right)$, systolic blood pressure (SBP), low-density lipoprotein cholesterol (LDL-C), and estimated glomerular filtration rate (eGFR) in patients with type 1 diabetes mellitus.

We constructed gradient boosting models to estimate the most important predictors for the selected risk factors HbA ${ }_{1 c^{\prime}} S B P, L D L-C$, and eGFR. The measure of strength of association in gradient boosting is denoted as relative influence. (A) $\mathrm{HbA}_{1 c^{\prime}}$ (B) SBP, (C) LDL-C, and (D) eGFR.

sociated with a significantly lower risk throughout for each of the outcomes.

Our analyses of optimal levels for $\mathrm{HbA}_{1 c^{\prime}} \mathrm{SBP}$, and LDL-C show that some of these risk factors display almost a linear relationship for risk of outcomes. This is clearest for $L D L-C$ in relation to $\mathrm{MI}$ and death and SBP in relation to death and $\mathrm{HF}$, for which levels lower than recommended by contemporary guidelines were associated with lower risk for outcomes. Although we accept that Cls do cross 1.0 in some cases, this is likely the result of limited power (Figure 4). The combination of proportional hazard models and machine learning analyses offers a unique and complementary ability to study these associations. Machine learning takes into account complex higher-order interactions and offers more accuracy and robustness, ${ }^{8-10}$ whereas the Cox models provide the ability to assess strength of association without permitting age to drown out the effect of other risk factors. The combination of statistical approaches suggests that the robust predictability of renal function and duration of diabetes mellitus may be integrated through (ie, in part explained by) age and other cardiometabolic risk factors, particularly by dysglycemia and SBP. The relative prognostic importance of $\mathrm{HbA}_{1 \mathrm{c}}$ is lower in the machine learning models compared with the Cox models, whereas albuminuria and SBP are prognostically more important in the machine learning models. This suggests that a significant interaction among age, $\mathrm{HbA}_{1 c^{\prime}}$ SBP, and albuminuria modifies the relative prognostic importance of these risk factors. Smoking demonstrated a low relative importance for all outcomes in our study, probably a result in part of the overall low prevalence of smoking.

Randomized trials have provided inconsistent evidence with regard to glycemic control and its relationship with macrovascular complications. ${ }^{11-14}$ The DCCT (Diabetes Control and Complications Trial) evaluated the effects of intensive glycemic control, and after years of follow-up in the EDIC study (Epidemiology of Diabetes Interventions and Complications), investigators observed a significant risk reduction for cardiovascular disease. ${ }^{11-15}$ Our study shows that for death, each $1-\mathrm{mmol} / \mathrm{mol}$ higher $\mathrm{HbA}_{1 \mathrm{c}}$ was associated with an $\approx 2 \%$ higher relative risk or $\approx 23 \%$ higher relative risk per $1 \%$ higher $\mathrm{HbA}_{1 c^{\circ}}$. The Cox model results also revealed that physical inactivity is an important predictor for stroke, whereas the machine learning models show the increasing prognostic importance of SBP, with heteroge- 

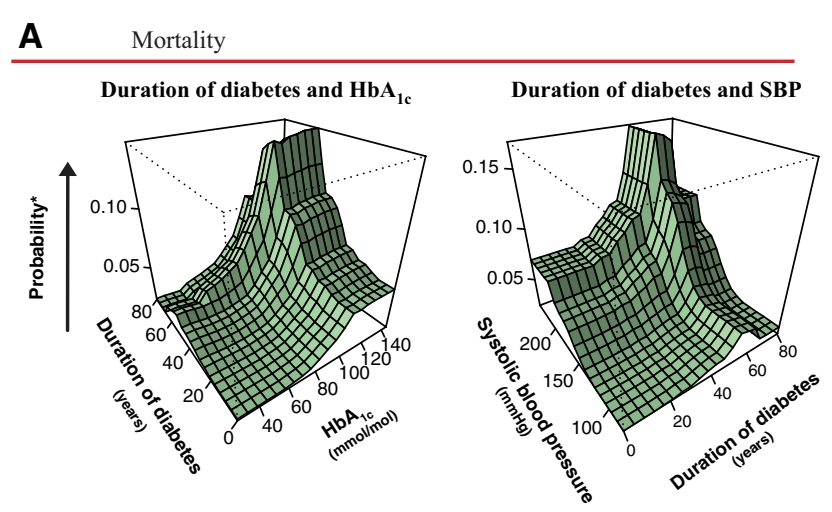

C Stroke
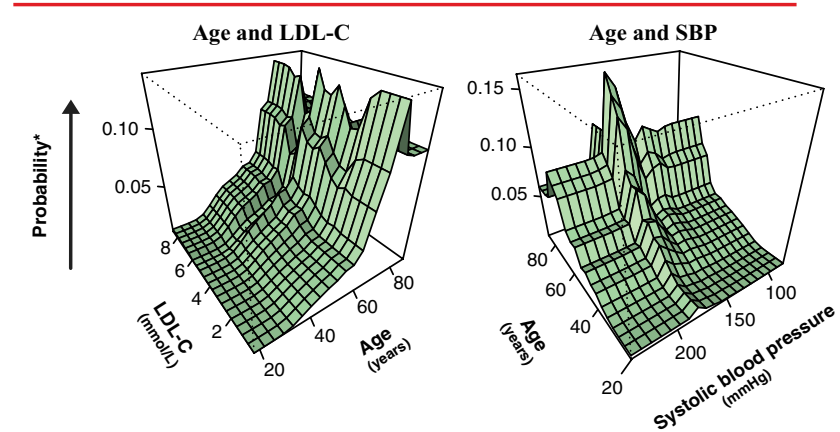

B

Acute myocardial infarction

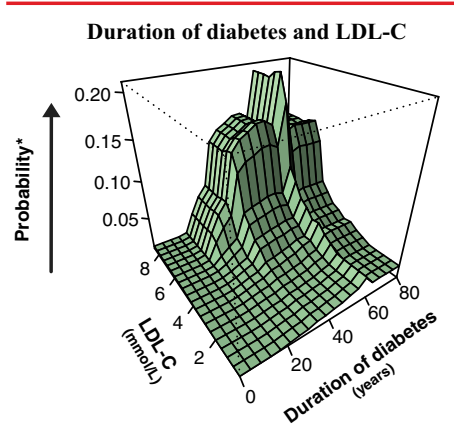

Duration of diabetes and SBP

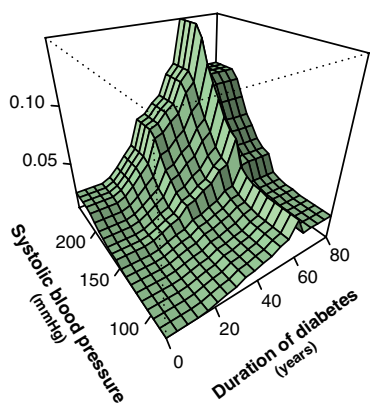

D Heart failure

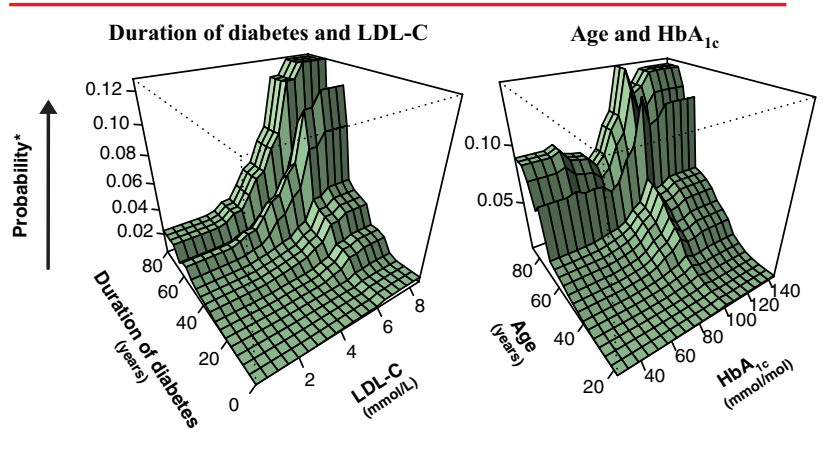

Figure 6. Gradient boosting for mortality and cardiovascular outcomes in patients with type 1 diabetes mellitus: partial dependence plots for the strongest predictors.

These 2-way partial dependence plots show the dependence between either age or duration of diabetes mellitus and 1 of the 5 most important predictors, marginalizing over the values of all other features. These are generated with gradient-boosting models for mortality and cardiovascular outcomes in patients with type 1 diabetes mellitus and enable us to visualize interactions among predictors. (A) All-cause mortality, (B) nonfatal/fatal acute myocardial infarction, (C) nonfatal/fatal stroke, and (D) hospitalization for heart failure. * The $y$ axis denotes the probability of an event during a 10.4-year period and ranges between 0 and 1.

neity of the association by varying age and degree of hyperglycemia. ${ }^{16}$

Awareness of cardiovascular disease risk in patients with hypercholesterolemia and T1DM has increased over time, yet contemporary data indicate that control on the population level remains suboptimal. ${ }^{17,18}$ For the present analyses, LDL-C was a strong predictor for $\mathrm{MI}$, stroke, and $\mathrm{HF}$, demonstrating $\approx 35 \%$ to $50 \%$ higher relative risk for each $1-\mathrm{mmol} / \mathrm{L}$ increase. Thus, patients with T1DM who have not developed cardiovascular disease, specifically younger patients, might benefit from more aggressive primary cardiovascular risk prevention efforts and perhaps efforts initiated earlier in the course of disease. ${ }^{18-21}$ This is an important clinical point because currently statins are less commonly used in patients with T1DM who are $<40$ years of age, ${ }^{22}$ and some clinicians remain unconvinced of the merits of statins in the care of their patients with T1DM despite some evidence. Our observational data showed strong associations between LDL-C and long-term cardiovascular risk in patients with T1DM (in particular, MI, with risks being lower at even lower levels), which adds important support for targeting LDL-C in T1DM to prevent vascular disease.

Previous studies claim that kidney dysfunction, in the form of microalbuminuria, macroalbuminuria, or reduced eGFR, fully accounts for the excess cardiovascular risk in T1DM. ${ }^{13,23}$ Our analyses imply that albuminuria is a strong risk factor for virtually all outcomes, associated with 2 to 4 times greater relative risk, with an overlap between various risk factors for albuminuria and cardiovascular disease, including hyperglycemia, hypertension, and hypercholesterolemia. Therefore, albuminuria is likely to represent an integrated estimate of several interrelated pathobiological mediators (ie, an integrated marker for the severity and duration of the perturbations of these risk factors). The present results suggest that intensive glycemic therapy may act through reducing microalbuminuria and macroalbuminuria and preserving eGFR, with findings from the DCCT directly supporting this notion. ${ }^{15}$

SBP, as expected, was a powerful predictor for cardiovascular disease, particularly for stroke. The long-term observations from the DCCT showed that higher $\mathrm{HbA}_{1 \mathrm{c}}$ was strongly associated with higher risk of hypertension, and intensive glycemic control reduced long-term risk of hypertension by $24 \% .{ }^{24}$ Other studies show that roughly $30 \%$ of patients with T1DM have hypertension and that hypertension is correlated with duration of diabetes mellitus, severity of hyperglycemia, and risk for development and progression of diabetic kidney disease. ${ }^{25,26}$ 
Studies focused on determining optimal levels of cardiovascular risk factors for patients with T1DM are scarce, with clinical context and recommendations somewhat extrapolated from randomized trials in type 2 diabetes mellitus. The present results show that lower $\mathrm{SBP}, \mathrm{HbA}_{1 c^{\prime}}$ and LDL-C levels are each associated with lower risk for cardiovascular outcomes and death, with a linear association for each with all outcomes assessed, except for mortality, for which $\mathrm{HbA}_{1 c}$ displayed a Ushaped association. Observational assessment of optimal levels is complicated because of reverse causality, residual confounding, and other factors limiting epidemiological data. Nevertheless, associations with outcomes were linear below levels presently recommended as guideline targets, and whether these targets should be more intensive in patients with T1DM should be assessed in future trials.

\section{Strengths and Limitations}

Virtually all patients with T1DM in Sweden are included in this observational study, with information available on comorbidities, medications, and risk factors. However, a minority of registrants had complete data available on all 17 characteristics assessed, requiring the use of imputation for missing data. An epidemiological definition of T1DM was used that may lack phenotypic precision, although this definition has been validated as highly accurate previously. Although both conventional and novel statistical approaches were used in our assessments of the strength of association of predictors adding complementary information, the results are model-dependent and could change slightly with different model building. We relied on baseline characteristics to model long-term outcomes, and it is likely that the results would be more accurate with time-updated information on risk factors during the period of observation. However, our approach mitigates to some degree the risk of reverse causation biasing the results. Finally, although numerous risk factors were accounted for in the adjusted models, it is not possible to completely overcome the limitation of residual confounding. A lower event number in those with risk factor levels at or below current target values means that $\mathrm{Cls}$ were inevitably wider. Therefore, some caution in interpretation is needed, although it is notable that patterns for associations for the 4 outcomes we examined in detail were broadly consistent.

\section{CONCLUSIONS}

Using data from a nationwide population-based cohort and state-of-the-art statistical methods, we found that the strongest predictors for cardiovascular outcomes and death are $\mathrm{HbA}_{1 c^{\prime}}$ albuminuria, duration of diabetes mellitus, LDL-C, and SBP during an average of 10 years of follow-up in individuals with T1DM. $\mathrm{HbA}_{1 \mathrm{c}}$ is a strong predictor for all outcomes, and its association is likely integrated with albuminuria and duration of diabetes mellitus, whereas LDL-C and SBP display independent predictability. LDL-C appears to be a more important prognostic factor than previously appreciated. Therefore, we believe that further research is warranted to delineate the potential benefit of more aggressive LDL-C lowering in patients with T1DM than currently practiced. The results also suggest a linear association for cardiovascular risk factors extending below thresholds used as contemporary treatment targets, and whether targeting lower levels may be clinically beneficial should be the focus of future trials.

\section{ARTICLE INFORMATION}

Received August 18, 2018; accepted January 14, 2019

Guest Editor for this article was David Maron, MD.

The online-only Data Supplement is available with this article at https://www. ahajournals.org/doi/suppl/10.1161/circulationaha.118.037454.

\section{Correspondence}

Aidin Rawshani, MD, PhD, Swedish National Diabetes Register, Region of Västra Götaland, Medicinaregatan 18G, 413 45, Gothenburg, Sweden. Email aidin. rawshani@gu.se

\begin{abstract}
Affiliations
Department of Molecular and Clinical Medicine, Institute of Medicine, University of Gothenburg, Sweden (A. Rawshani, A. Rawshani, B.E., A. Rosengren, S.G.). Swedish National Diabetes Register, Center of Registers in Region, Gothenburg, Sweden (A. Rawshani, A. Rawshani, S.F., B.E., A.-M.S., M.M., S.G.). Health Metrics Unit, Sahlgrenska Academy, University of Gothenburg, Sweden (S.F.). Institute of Cardiovascular and Medical Sciences, University of Glasgow, UK (N.S.). Department of Public Health and Caring Sciences/Geriatrics, Uppsala University, Sweden (B.Z.). Division of Cardiology, Department of Internal Medicine, University of Texas Southwestern Medical Center, Dallas (D.K.M.).
\end{abstract}

\section{Sources of Funding}

This work was supported by grants from the Swedish Heart and Lung Foundation (grant number 2017-0839), the Swedish Association of Local Authorities and Regions, the Swedish State under the agreement concerning research and education of doctors, Region Västra Götaland, the Swedish Diabetes Foundation, the Swedish Research Council (2013-5187, SIMSAM), and Diabetes Wellness Sweden.

\section{Disclosures}

None.

\section{REFERENCES}

1. Rawshani A, Rawshani A, Franzén S, Eliasson B, Svensson AM, Miftaraj M, McGuire DK, Sattar N, Rosengren A, Gudbjörnsdottir S. Mortality and cardiovascular disease in type 1 and type 2 diabetes. $N$ Engl J Med. 2017;376:1407-1418. doi: 10.1056/NEJMoa1608664

2. Livingstone SJ, Levin D, Looker HC, Lindsay RS, Wild SH, Nicola J, Leese G, Leslie P, McCrimmon RJ, Metcalfe W, McKnight JA, Morris AD, Pearson D, Petrie J, Philip S, Sattar N, Traynor JP, Colhoun H. Estimated life expectancy in a Scottish cohort with type 1 diabetes, 2008-2010. JAMA. 2015;313:37-44. doi:10.1001/jama.2014.16425

3. Rawshani A, Rawshani A, Franzén S, Eliasson B, Svensson AM, Miftaraj M, McGuire DK, Sattar N, Rosengren A, Gudbjörnsdottir S. Range of risk factor levels: control, mortality, and cardiovascular outcomes in type 1 diabetes mellitus. Circulation. 2017;135:1522-1531. doi: 10.1161/CIRCULATIONAHA.116.025961 
4. Lind $M$, Svensson AM, Kosiborod M, Gudbjörnsdottir S, Pivodic $A$ Wedel H, Dahlqvist S, Clements M, Rosengren A. Glycemic control and excess mortality in type 1 diabetes. N Eng/ J Med. 2014;371:1972-1982. doi: 10.1056/NEJMoa1408214

5. Eliasson B, Gudbjörnsdottir S. Diabetes care: improvement through measurement. Diabetes Res Clin Pract. 2014;106(suppl 2):S291-S294. doi: 10.1016/S0168-8227(14)70732-6

6. Ludvigsson JF, Andersson E, Ekbom A, Feychting M, Kim JL, Reuterwall C, Heurgren M, Olausson PO. External review and validation of the Swedish National Inpatient Register. BMC Public Health. 2011;11:450. doi: 10.1186/1471-2458-11-450

7. Heller G. A measure of explained risk in the proportional hazards model. Biostatistics. 2011;13:315-325. doi:10.1093/biostatistics/kxr047

8. Ishwaran H, Kogalur UB. R-Package: Random Forest for Survival, Regression and Classification (RF-SRC). Vienna, Austria: R Foundation for Statistical Computing; 2018. R-package version 1.6.1.

9. Ridgeway G. gbm: Generalized boosted regression models: R-package version 2.1.1. 2015. http://CRAN.R-project.org/package=gbm. Accessed March 28, 2019.

10. Liaw A, Wiener M. Classification and regression by randomForest: R-package. $R$ News. 2002;2:18-22.

11. Lehto S, Rönnemaa T, Pyörälä K, Laakso M. Poor glycemic control predicts coronary heart disease events in patients with type 1 diabetes without nephropathy. Arterioscler Thromb Vasc Biol. 1999;19:1014-1019.

12. Soedamah-Muthu SS, Chaturvedi N, Witte DR, Stevens LK, Porta M, Fuller JH; EURODIAB Prospective Complications Study Group. Relationship between risk factors and mortality in type 1 diabetic patients in Europe: the EURODIAB Prospective Complications Study (PCS). Diabetes Care. 2008:31:1360-1366. doi: 10.2337/dc08-0107

13. Orchard TJ, Secrest AM, Miller RG, Costacou T. In the absence of renal disease, 20 year mortality risk in type 1 diabetes is comparable to that of the general population: a report from the Pittsburgh Epidemiology of Diabetes Complications Study. Diabetologia. 2010;53:2312-2319. doi: 10.1007/s00125-010-1860-3

14. Orchard TJ, Olson JC, Erbey JR, Williams K, Forrest KY, Smithline Kinder L, Ellis D, Becker DJ. Insulin resistance-related factors, but not glycemia, predict coronary artery disease in type 1 diabetes: 10-year follow-up data from the Pittsburgh Epidemiology of Diabetes Complications Study. Diabetes Care. 2003;26:1374-1379.

15. Nathan DM, Bayless M, Cleary P, Genuth S, Gubitosi-Klug R, Lachin JM, Lorenzi G, Zinman B; DCCT/EDIC Research Group. Diabetes control and complications trial/epidemiology of diabetes interventions and complications study at 30 years: advances and contributions. Diabetes. 2013;62:3976-3986. doi: 10.2337/db13-1093

16. Nathan DM, Cleary PA, Backlund J-YC, DCCT/EDIC Study Research Group. Intensive diabetes treatment and cardiovascular disease in patients with type 1 diabetes. N Engl J Med. 2005;353:2643-2653. doi:10.1056/NEJMoa052187

17. Orchard TJ, Forrest KY, Kuller LH, Becker DJ; Pittsburgh Epidemiology of Diabetes Complications Study. Lipid and blood pressure treat- ment goals for type 1 diabetes: 10-year incidence data from the Pittsburgh Epidemiology of Diabetes Complications Study. Diabetes Care. 2001:24:1053-1059.

18. Hero C, Rawshani A, Svensson AM, Franzén S, Eliasson B, Eeg-Olofsson K, Gudbjörnsdottir S. Association between use of lipid-lowering therapy and cardiovascular diseases and death in individuals with type 1 diabetes. Diabetes Care. 2016;39:996-1003. doi: 10.2337/dc15-2450

19. Cholesterol Treatment Trialists' (CTT) Collaboration, Fulcher J, O'Connell R. Efficacy and safety of LDL-lowering therapy among men and women: metaanalysis of individual data from 174,000 participants in 27 randomised trials. Lancet. 2015;385:1397-1405. doi:10.1016/50140-6736(14)61368-4

20. Burgess $S$, Ference $B A$, Staley JR, Freitag $D F$, Mason AM, Nielsen SF, Willeit P, Young R, Surendran P, Karthikeyan S, Bolton TR, Peters JE, Kamstrup PR, Tybjaerg-Hansen A, Benn M, Langsted A, Schnohr P, Vedel-krogh S, Kobylecki CJ, Ford I, Packard C, Trompet S, Jukema JW, Sattar N, Di Angelantonio E, Saleheen D, Howson JM, Nordestgaard BG, Butterworth AS, Danesh J; European Prospective Investigation Into Cancer and Nutrition-Cardiovascular Disease (EPIC-CVD) Consortium. Association of LPA variants with risk of coronary disease and the implications for lipoprotein(a)-lowering therapies: a mendelian randomization analysis. JAMA Cardiol. 2018;3:619-627. doi:10.1001/jamacardio.2018.1470

21. Zgibor JC, Wilson RR, Orchard TJ. Has control of hypercholesterolemia and hypertension in type 1 diabetes improved over time? Diabetes Care. 2005;28:521-526.

22. Livingstone SJ, Looker HC, Hothersall EJ, Wild SH, Lindsay RS, Chalmers J, Cleland S, Leese GP, McKnight J, Morris AD, Pearson DWM, Peden NR, Petrie JR, Philip S, Sattar N, Sullivan F, Colhoun HM. Risk of cardiovascular disease and total mortality in adults with type 1 diabetes: Scottish Registry Linkage Study. PLoS Med. 2012;9:e1001321. doi:10.1371/journal.pmed. 1001321

23. Groop PH, Thomas MC, Moran JL, Wadèn J, Thorn LM, Mäkinen VP, Rosengård-Bärlund M, Saraheimo M, Hietala K, Heikkilä O, Forsblom C; FinnDiane Study Group. The presence and severity of chronic kidney disease predicts all-cause mortality in type 1 diabetes. Diabetes. 2009;58:16511658. doi: $10.2337 / \mathrm{db} 08-1543$

24. de Boer $I H$, Kestenbaum B, Rue TC, Steffes MW, Cleary PA, Molitch ME, Lachin JM, Weiss NS, Brunzell JD; Diabetes Control and Complications Trial (DCCT)/Epidemiology of Diabetes Interventions and Complications (EDIC) Study Research Group. Insulin therapy, hyperglycemia, and hypertension in type 1 diabetes mellitus. Arch Intern Med. 2008;168:1867-1873. doi: 10.1001/archinternmed.2008.2

25. Chillarón JJ, Sales MP, Flores-Le-Roux JA, Murillo J, Benaiges D, Castells I, Goday A, Cano JF, Pedro-Botet J. Insulin resistance and hypertension in patients with type 1 diabetes. J Diabetes Complications. 2011;25:232236. doi: 10.1016/j.jdiacomp.2011.03.006

26. Collado-Mesa F, Colhound HM, Stevens LK, Boavida J, Ferriss JB, Karamanos B, Kempler P, Michel G, Roglic G, Fuller JH. Prevalence and management of hypertension in type 1 diabetes mellitus in Europe: the EURODIAB IDDM Complications Study. Diabetes Med. 1998:1-8. doi.org/10.1046/j.1464-5491.1999.00007.x 